\title{
A fatal postpartum Clostridium sordellii associated toxic shock syndrome
}

\author{
A Bitti, P Mastrantonio, P Spigaglia, G Urru, A I Spano, G Moretti, G B Cherchi
}

\begin{abstract}
Clostridium sordellii is an infrequent human pathogen. It has been demonstrated to be occasionally responsible for myonecrosis or gas gangrene. Interestingly, in the obstetric literature, some cases of postpartum maternal deaths have been associated with $C$ sordellii infection causing a rapidly lethal toxin mediated syndrome. This is the first reported case of postpartum death in a 29 year old woman, in which a toxigenic $C$ sordellii was isolated from the patient's blood antemortem during the fatal toxic shock, strongly indicating its role in this rare syndrome.

$(\mathcal{F}$ Clin Pathol 1997;50:259-260)
\end{abstract}

Keywords: Clostridium sordellii; shock; toxin; postpartum.

Clostridium sordellii is a Gram positive rod and an obligate anaerobe. It is known as an aetiological agent of enteritis and enterotoxaemia in sheep and cattle, ${ }^{1}$ of myonecrosis or gas gangrene in humans, and of neonatal toxin omphalitis. ${ }^{2}$ The haemorrhagic and lethal toxins produced by $C$ sordellii are related to Clostridium difficile toxins $\mathrm{B}$ and $\mathrm{A}$, respectively, ${ }^{3}$ and are responsible for refractory shock observed in some animal and human infections. In the obstetric literature, fatal cases of infections involving toxigenic $C$ sordellii have rarely been described. ${ }^{45}$ The clinical aspects of these cases included: sudden onset with an influenza-like prodrome, progressive refractory hypotension, hypothermia or absence of fever, and absence of purulent discharge. Laboratory clinical parameters included notable leukaemoid reaction, and elevated haematocrit, findings of mild disseminated intravascular coagulopathy, abnormal results of urine analysis, decreased serum calcium and increased liver enzymes and creatinine values. Distinctive pathological findings included significant local and spreading oedema with pleural and peritoneal effusion, localised tissue necrosis, notable inflammatory reaction limited to the margins of necrotic tissue, and thrombosis of nearby vessels.

This paper reports a case of postpartum death of a $C$ sordellii associated toxic shock syndrome, in which a urease negative $C$ sordellii strain was isolated from the blood of the 29 year old patient. This is the first case of postpartum death in which a toxigenic $C$ sordellii was isolated from the patient's blood during the fatal toxic shock, strongly indicating its implication in this syndrome.

\section{Case report}

A caesarean section was performed in a 29 year old woman, gravida 1 , for cervical dystocia in the 41 st week. The postoperative course was normal. Two days after delivery the patient was afebrile but complained of generalised weakness, dizziness in an orthostatic position, and pain in the epigastric region followed by hypotension $(80 / 50 \mathrm{~mm} \mathrm{Hg})$, sinus tachycardia ( 110 beats/minute), and low urinary output. The patient was given fluids and steroids but hypotension proved refractory. The abdomen was painful and tender. Ultrasonogram showed hyperperistalsis and anechoic areas scattered in the abdominal cavity; blood analysis showed noticeable haemoconcentration and hyperproteinemia. Laparotomy revealed a substantial amount of serosanguineous fluid, the peritoneum was lifted by a jelly-like retroperitoneal tissue, the appendiceal apex was hyperaemic and adhered to the rear parietal peritoneum, and the right adnexa appeared oedematous and thickened. Gall bladder, duodenum, stomach, ileum, ascendens, and transverse colon appeared thickened and ischaemic. Pancreas, spleen, and liver were normal. The patient underwent appendectomy and removal of the right adnexa, and was treated with antibiotics (intravenous cefotaxime $1 \mathrm{~g}$ three times a day.). Peritoneal exudate and blood were collected and cultured. Although clinical conditions partially improved, after a few hours the hypotension became refractory, her skin was pale and cool with cyanotic extremities, her temperature was normal, haematocrit was $57.6 \%$, haemoglobin was $246 \mathrm{~g} / \mathrm{l}$, white cell count was $115 \times$ $10^{9} / 1$; a decrease in total protein concentration $(26 \mathrm{~g} / \mathrm{l})$, and calcium concentration $(64 \mathrm{mg} / \mathrm{l})$ was also observed. Because of serious cardiac conditions the patient was moved to an intensive care unit where she died six hours later.

\section{Microbiological studies}

Blood was collected during laparotomy while the patient was on antibiotic therapy. Bactec 26A and Bactec 27A broths (Becton Dickinson Italia, Milan) were used to grow aerobes and anaerobes, respectively, in an automatic Bactec NR 730. Subcultures were performed using Schaedler agar (Difco Laboratories, Detroit, Michigan) supplemented with vitamin $\mathrm{K}$ for anaerobes, and mannitol salt agar, desoxycholate agar, Colombia blood agar and Sabouraud agar (Difco) for aerobes and yeasts. The peritoneal fluid was inoculated into thioglycollate and tryptose broth (Difco). The assay for detection of toxic shock syndrome toxin 1 (TSST-1) of Staphylococcus aureus on the 00161 Rome, Italy. 
patient's serum ${ }^{6}$ was not performed as the patient did not present the typical clinical manifestations linked to TSST-1 such as fever and erythematous rash. ${ }^{7}$ After 24 hours of incubation, the Bactec 27A broth proved positive and the subsequent subculture in Schaedler agar, incubated in anaerobic conditions, showed the presence of greyish, irregular colonies. Gram stain showed Gram positive bacilli with terminal and free spores.

Subcultures on Columbia blood agar supplemented with $0.1 \%$ vitamin $\mathrm{K}$ and $5 \%$ yeast extract were used for identification by Rapid ID 32A and the API20A kits (bioMériex, Marcy l'Etoile). The negative result of the urease test made the discrimination between $C$ sordellii and Clostridium bifermentans difficult. This uncertain result prompted further investigation by gasliquid chromatography analysis of methylated non-volatile fatty acids. ${ }^{8}$ The absence of succinic acid confirmed the strain as $C$ sordellii.

The $C$ sordellii cytotoxicity was assayed on Chinese Hamster Ovary Cells (CHO) by the microtitre method. ${ }^{9}$ One millilitre of an overnight culture in Cooked meat (Becton Dickinson Microbiology System, Cockeysville, Maryland) supplemented with $10 \%$ glucose was centrifuged at $17,000 \times g$ for 15 minutes. A $20 \mu \mathrm{l}$ aliquot of an undiluted filtered supernatant and of twofold serially diluted samples was added to each well with a confluent monolayer of cells. The plate was incubated at $37^{\circ} \mathrm{C}$ for 24 hours. For the toxin neutralisation test, an equal volume of $C$ sordellii antitoxin (VPI, Blacksburg, Virginia) was added to the supernatant preincubated at $37^{\circ} \mathrm{C}$ for one hour and then added to each well, as described earlier. The culture filtrate was cytopathic for the $\mathrm{CHO}$ monolayer. The cells were rounded up to a titre of 1:64. The $C$ sordellii antitoxin completely neutralised the cytotoxic activity of the sample.

Lethal toxicity of the $C$ sordellii filtrate was assayed on six-week-old BALB/c mice. Three filtrate dilutions $(0.5 \mathrm{ml}$ of $1: 10,1: 50$, or $1: 100$ ) in $0.05 \mathrm{M}$ phosphate buffer, $\mathrm{pH} 6.5$, were injected intraperitoneally into five mice per dilution. The ability of the antitoxin to neutralise the lethal toxicity of the filtrate was determined by incubating a mixture of an equal volume of $1: 100$ dilution of the filtrate and $C$ sordellii antitoxin for an hour at $37^{\circ} \mathrm{C}$. After incubation, $0.5 \mathrm{ml}$ of the mixture was injected intraperitoneally into five mice. A noninoculated medium filtrate and a 1:100 dilution of $C$ sordellii toxin were used as negative and positive controls, respectively. The lethal toxicity assay showed that all mice inoculated with the culture filtrate up to $1: 100$ dilution died after 24 hours, whereas those inoculated with the sample preincubated with $C$ sordellii antitoxin survived. The culture of the patient's peritoneal fluid was negative.

\section{Discussion}

$C$ sordellii is a rare human pathogen. This microorganism produces an oedema producing toxin and a haemorrhagic toxin. The former is more lethal than the latter. ${ }^{9}$ Both toxins are related to $C$ difficile toxins $\mathrm{B}$ and $\mathrm{A}$, respectively. ${ }^{3}$ Few human $C$ sordellii infections, with demonstrated or suspected toxin production, have been documented in literature..$^{24510}$ In 1989 McGregor et al summarised clinical features and laboratory findings of $C$ sordellii associated shock syndrome in three postpartum maternal deaths. ${ }^{11}$

This case report describes a healthy woman who died postpartum due to a toxic shock syndrome caused by a toxigenic $C$ sordellii strain. To our knowledge, this is the first report in which the microorganism was isolated from the patient's blood during the shock. Laboratory experiments showed that $C$ sordellii antiserum neutralised the toxicity of this microorganism both in vitro and in vivo. Clostridial infections generally require an early diagnosis, and combined medical and surgical treatment. Effective therapy is thus even more difficult in $C$ sordellii infections leading to a shock syndrome, because of the scant experience, and extremely rapid clinical course. Moreover, bacterial identification can be complicated by the unusual biochemical characteristics of this bacterium, such as the negative urease test in the present case when the microorganism may be misclassified as $C$ bifermentans, which is not a toxin producer. ${ }^{12}$

Antibiotic treatment should include agents with significant activity against the microorganism such as penicillin, tetracycline, metronidazole, clindamycin, and imipenem, ${ }^{13}$ but antitoxin serum might be more rapid and specific.

$C$ sordellii and $C$ difficile antisera are known to be effective in preventing illness and death in animal models. ${ }^{14}$ Although therapy with $C$ sordellii or $C$ difficile antitoxin has not been experimented in humans, it may be useful for $C$ sordellii toxaemia cases, and perhaps could save lives.

Borriello SP. Clostridia in gastrointestinal disease. Boca Raton: CRC Press, Inc, 1985.

2 Adamkiewicz TV, Goodman D, Burke B, Lyerly DM, Goswitz J, Ferrieri P. Neonatal Clostridium sordellii toxic omphalitis. Pediatr Infect Dis f 1993;12:253-7.

3 Martinez RD, Wilkins TD Comparison of Clostridium sordellii toxins $\mathrm{HT}$ and $\mathrm{LT}$ with toxins $\mathrm{A}$ and $\mathrm{B}$ of $C$. difficile. s Med Microbiol 1992;36:30-6.

4 McGregor JA, Soper DE, Lovell G, Todd JK. Maternal deaths associated with Clostridium sordellii infection. Am $f$ Obstet Gynecol 1989;161:987-95.

$5 \mathrm{Hogan} \mathrm{SF}$, Ireland K. Fatal acute spontaneous endometritis resulting from Clostridium sordellii. Am $\mathcal{f}$ Clin Pathol 1989;91:104-6.

6 Miwa K, Fukuyama M, Kunitomo T, Igarashi H. Rapid assay for detection of toxic shock syndrome toxin 1 from human sera. 7 Clin Microbiol 1994;32:539-42.

7 Todd J, Fishuat $M$, Kapral F, Welch T. Toxic shock syndrome associated with phage-group-1 staphylococci. Lancet. 1978;ii:1116-8.

8 Summanen P, Baron EJ, Citron DM, Strong CA, Wexler HM, Finegold SM. Wadsworth anaerobic bacteriology manual. Belmont: Star Publishing Company, 1993.

9 Nakamura S, Ogura H, Tanaka J, Tanabe N, Yamakawa K, Nakamura S, Ogura H, Tanaka J, Tanabe N, Yamakawa $\mathrm{K}$,
Hatano M, et al. Difference in susceptibility of various cell culture to cytotoxic culture filtrates of Clostridium sordellii.

Microbiol Immunol 1984;28:493-7.
10 Arseculeratne SN, Panabokke RG, Wijesundera S. The toxins responsible for the lesions of Clostridium sordellii gas gangrene. $\mathcal{F}$ Med Microbiol 1969;2:37-53.

11 Spera RV, Mark HK. Clostridium sordellii bacteremia: case report and review. Clin Infect Dis 1992;15:950-4.

2 Nakamura S, Shimamura T, Nishida S. Urease-negative strains of Clostridium sordellii. Can $\mathcal{F}$ Microbiol 1976;22:673-

13 Nakamura S, Yamakawa K, Nishida S. Antibacterial susceptibility of Clostridium sordellii strains. Zentralbl Bakt Hy 1986;261:345-9.

$14 \mathrm{Kim}$ PH, Iaconis JP, Rolfe RD. Immunization of adult hamsters against Clostridium difficile-associated ileocecitis and transfer of protection to infant hamster. Infect Immun 1987; 55:2984-92. 